\title{
Alpha-Smooth Muscle Actin (a-SMA) Gene, a Possible Derive of Myofibroblasts in Bone Marrow Fibrosis
}

\author{
Amira Osama ${ }^{\mathrm{a}}$, Amal El-Mahdy ${ }^{\mathrm{a}}$, Samea Rizk ${ }^{\mathrm{b}}$, Howayda Mohamed ${ }^{\mathrm{a}}$, Hanan El-Hosseiny ${ }^{\mathrm{b}}$, \\ Manal Wilson ${ }^{\mathrm{b}}$, Amira Noureldin ${ }^{\mathrm{a}}$
}

a

Department of Clinical and Chemical Pathology, Benha Faculty Of Medicine, Benha university, $\quad{ }^{\mathrm{b}}$ Department of Clinical and Chemical Pathology, Cairo Faculty of medicine, Cairo University, Egypt.

Correspondence to:

Amira Osama, Department of Clinical and Chemical Pathology, Benha Faculty Of Medicine, Benha university,

Email:

amosmady@yahoo.com

Received: 9 October 2019

Accepted: 17 March 2020

\begin{abstract}
:
Background: Alpha-smooth muscle actin ( $\alpha-\mathrm{SMA})$ is used as a marker for a subset of activated fibrogenic cells, myofibroblasts, which are regarded as important effector cells of tissue fibrogenesis. Purpose: We addressed whether ASMA gene (actin alpha 2 gene or ACTA2) expression is up regulated in cases with $\mathrm{BM}$ fibrosis compared to those with no bone marrow (BM) fibrosis in order to evaluate its role in the pathogenesis of BM fibrosis. Subjects and methods: ASMA expression was detected by quantitative RT-PCR in formalin fixed paraffin embedded (FFPE) bone marrow trephine biopsy samples of cases with neoplastic bone marrow diseases as well as reactive bone marrow disorders cases. Both groups included cases with and without bone marrow fibrosis. Results: Results indicated that there was no statistically significant difference in ASMA (ACTA2) gene expression between neoplastic fibrotic and non-fibrotic cases as well as between reactive fibrotic and non-fibrotic cases. Also the level of $\alpha$-SMA expression does not correlate positively with the grade of bone marrow fibrosis. Conclusion: We conclude that $\alpha$ -
\end{abstract} SMA is not a functional marker of fibrogenic cells in bone marrow fibrosis. Exploration of other related genetic pathway is recommended.

Key words: Alpha-smooth muscle actin, Bone marrow fibrosis, Myofibroblast. 



\section{Introduction:}

Bone marrow (BM) fibrosis is the continuous replacement of blood-forming cells in the BM by excessive scar tissue, leading to failure of the body to produce blood cells and ultimately to death (1).

A number of haematological and nonhaematological disorders are associated with increased BM fibrosis, including myelodysplastic syndromes and myeloproliferative neoplasms (MPNs) (2).

Fibrosis is a wound healing response to injury and characterized by excessive synthesis and accumulation of extracellular matrix (ECM) proteins in many organs such as skin, liver, lung, kidney, and heart, which ultimately leads to organ failure and death (3).

Pathologically, myofibroblasts are the major cellular source of ECM in most forms of fibrogenesis, despite the fact that myofibroblasts may originate from various cell types depending on the organ and mechanism of injury (4).

Nevertheless, myofibroblasts share two common features de novo expression of smooth muscle alpha-actin (SM a-actin) and abundant ECM, particularly type 1 collagen A1 (COL1A1) (5).
Actins consist of six isoforms and play an essential role in many cell processes, including cell proliferation, differentiation, migration, and contraction (6)

The Actin Alpha 2 (ACTA2) gene encoding smooth muscle aortic actin, was isolated and characterized. It was found that the ACTA2 gene contains at least 9 exons (7).

Alpha smooth muscle actin appears to play an important functional role in myofibroblast contraction and migration. Previous studies have shown that stress fibers containing SM a-actin, not only generate greater contractile force than stress fibers that contain only cytoplasmic-b and gactin, but also associate with super mature focal adhesions, which in turn exhibit a specific molecular composition of integrins avb3 and a5b1 (8) (9).

Integrins are transmembrane proteins, and thus integrins can bidirectionally transmit the mechanical signal across the cell membrane (10).

Although the molecular mechanisms by which the mechanical signal, transmitted by integrins associated with $\alpha$-SMA, regulates extracellular matrix expression remain 
unclear. Studies have revealed that the cell contractile force (mediated by actin stress fibers), as a mechanical signal, could be transformed to a chemical signal to modulate ECM genes such as COL1A1 expression through transforming growth factor-b (TGF-b) and Erk signal pathways (11).

Although multiple evidence supports that ASMA plays an important role in multiple features of myofibroblasts, its role in fibrogenesis remains controversial (12).

To shed light on this question, our study evaluated the degree of ACTA2 gene expression in cases with $\mathrm{BM}$ fibrosis compared to those with no BM fibrosis in order to evaluate its role in the pathogenesis of BM fibrosis and thus defining patients that could potentially benefit from ASMAtargeted therapy.

\section{Subjects and methods:}

\section{- Study design and protocol}

This case control study included collection of archived formalin fixed- paraffin embedded bone marrow trephine biopsies of patients with different underlying diseases from the bone marrow pathology unit, Clinical pathology department, Faculty of medicine, Cairo University during the period from June 2016 to October 2018.
Participants gave their informed written consent before enrolment and the study was approved by the Research Ethics Committee in Faculty of Medicine, Benha University.

RNAs of 90 adequate samples were used to complete the study. Those included 45 cases with neoplastic bone marrow diseases and 45 cases with reactive bone marrow disorders.

\section{- Key inclusion criteria}

Any archived bone marrow core biopsy that fulfils the fixation and processing requirements for the molecular study.

\section{- Key exclusion criteria}

We excluded bad archived trephine bone marrow biopsy samples and those which were not adequately processed.

\section{- Methodology}

Cases of different underlying pathology are subjected to the following laboratory techniques:

1- Provisional selection of the samples $\underline{\text { with full data recording }}$

2- Bone marrow samples were stained for reticulin (Silver impregnation) according to (13) \& trichrome according to (14) Evaluation of fibrosis grade is assessed according to the 2016 WHO grading of BM fibrosis (15). 
According to the underlying bone marrow conditions cases were divided into 2 groups:

Group 1: Neoplastic group that included 45 cases with neoplastic bone marrow disorders (acute myeloid leukemia (AML), myelodysplastic syndrome (MDS), myeloproliferative neoplasms (MPNs), lymphoproliferative and plasma cell disorders).

Group 2: Reactive group that included 45 cases with normal and reactive bone marrow changes (included cases of erythroid hyperplasia, hypercellular marrow with eosinophila and increased megakaryocytes and sarcoidosis) as well as cases with miscellaneous conditions.

According to the bone marrow fibrotic status both groups were further divided into 2 subgroups using the 2016 WHO grading of BM fibrosis Fibrotic subgroup included cases with bone marrow collagen fibrosis grade $\geq 1$.

Non-fibrotic subgroup included cases with < grade 1 collagen fibrosis.

The neoplastic group included 26 (57.8\%) trephine bone marrow samples belonging to fibrotic subgroup and 19
(42.2\%) belonging to non-fibrotic subgroup. While the reactive group included 17 (37.7\%) samples belonging to fibrotic subgroup and $28(62.3 \%)$ samples belonging to non-fibrotic subgroup.

\section{3- Real-time PCR.}

Total RNA was extracted from bone marrow trephine biopsy samples using ready-made commercial kit (RNeasy FFPE Kit, cat no. 73504) according to the manufacturer's protocol. Isolated RNA samples were then used for RT-PCR. Samples of total RNA (1 $\mu \mathrm{g})$ were reverse transcribed into cDNA in $20 \mu \mathrm{l}$ reaction volumes using a first-strand cDNA synthesis kit for RT-PCR according to the manufacturer's instructions (MBI Fermentas, Hanover, MD, USA). Gene expression levels were quantified by real-time PCR using a SensiMix SYBR kit (Bioline Ltd., London, UK) and $100 \mathrm{ng}$ of cDNA per reaction. The primer sequences used for this analysis were as follows: human $\alpha$-SMA (forward, 5'-AGA CAT CAG GGG GTG ATG GT-3' and reverse, 5'-CAT GGC TGG GAC ATT GAA AG-3'), and GAPDH was used as an internal control (forward, 5'-ATT GTT GCC ATC AAT GAC CC-3' and reverse, 5'-AGT AGA GGC AGG GAT GAT GT-3'). An annealing temperature of $60^{\circ} \mathrm{C}$ was used for all the 
primers. PCR was performed in a standard 96-well plate format with an ABI 7500HT Real-Time PCR Detection System (Thermo

Fisher Scientific, Inc.). For data analysis, the raw threshold cycle (CT) value was first normalized to the housekeeping gene for each sample to obtain a $\Delta \mathrm{CT}$.

\section{Statistical analysis:}

Data management and statistical analysis were done using SPSS v.25 (IBM, Armonk, New York, U.S.A). Numerical data were summarized using mean and standard deviation or median and range. Categorical data was summarized using numbers and percentage.

Different levels of gene expression were compared using Mann-Whitney test. Correlation analysis was done between different levels of gene expression and degree of reticulin bone marrow fibrosis using spearman correlation. " $r$ " is the correlation coefficient. It ranges from -1 to +1. -1 indicates strong negative correlation. +1 indicates strong positive correlation. All $\mathrm{P}$ values were two sided. $\mathrm{P}$ values less than 0.05 were considered significant.

\section{Results:}

Baseline characteristics of the study population was shown in Table $1 \&$ Figure 1

\begin{tabular}{lllll}
\hline & \multicolumn{2}{c}{ Neoplastic } & $(\mathbf{4 5}$ cases) & \multicolumn{2}{c}{ Reactive (45 cases) } \\
& Fibrotic & $\begin{array}{l}\text { Non- } \\
\text { fibrotic }\end{array}$ & Fibrotic & $\begin{array}{l}\text { Non- } \\
\text { fibrotic }\end{array}$ \\
\hline $\begin{array}{l}\text { Number } \\
(\%)\end{array}$ & 26 & 19 & 17 & 28 \\
$(57.8 \%)$ & $(42.2 \%)$ & $(37.7 \%)$ & $(62.3 \%)$ \\
\hline
\end{tabular}

Table 1.:Number and percentages of cases in each subgroup

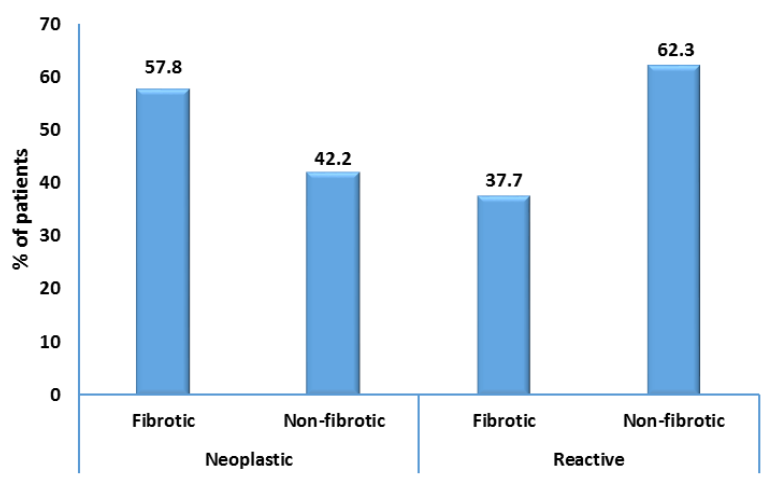

Figure 1. Number and percentage of the fibrotic and non-fibrotic subgroups in each of the neoplastic and reactive groups.

Gene expression findings

On comparing fibrotic versus non-fibrotic subgroups in the neoplastic as well as in the reactive groups median ACTA2 gene expression showed no statistically significant difference $(\mathrm{P}$ value $=0.819,1.00$ respectively) Table $\mathbf{2}$, fig 2,3. 
No statistically significant correlations were and the grade of fibrosis in all studied cases detected between ACTA2 gene expression table 3 .

Table 2. . Comparison of ACTA2 gene expression between fibrotic and non-fibrotic subgroups in both neoplastic and reactive groups.

\begin{tabular}{cllllll}
\hline & Neoplastic (45 cases) & $\begin{array}{l}\text { P } \\
\text { value }\end{array}$ & Reactive (45 cases) & $\begin{array}{l}\text { P } \\
\text { value }\end{array}$ \\
\hline & Fibrotic & Non-fibrotic & Fibrotic & Non-fibrotic & \\
& $(26$ cases $)$ & $(19$ cases $)$ & & $(17$ cases $)$ & $(28$ cases $)$ & \\
& 0.07 & 0.03 & 0.814 & 0.02 & 0.03 & 1.00 \\
ACTA2 & $(0.00-0.49)$ & $(0.01-0.05)$ & & $(0.02-0.08)$ & $(0.00-0.04)$ & \\
Median & & & & & & \\
\hline
\end{tabular}

Table 3. Correlation between the degree of bone marrow fibrosis and ACTA2 gene expression in all studied cases.

\begin{tabular}{lll}
\hline & Spearman's rho & P value \\
\hline ACTA2 gene & -.538 & $\mathbf{0 . 2 1 3}$ \\
\hline
\end{tabular}

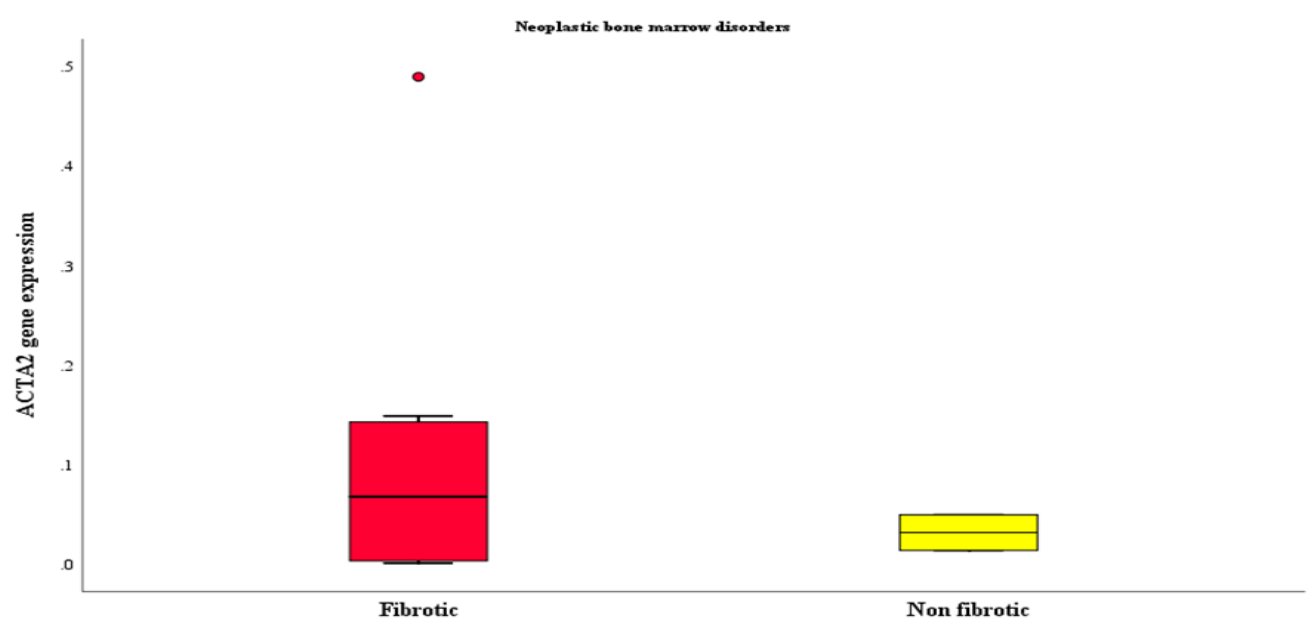

Figure 2: ACTA2 gene expression in fibrotic and non-fibrotic subgroups in the neoplastic groups. 


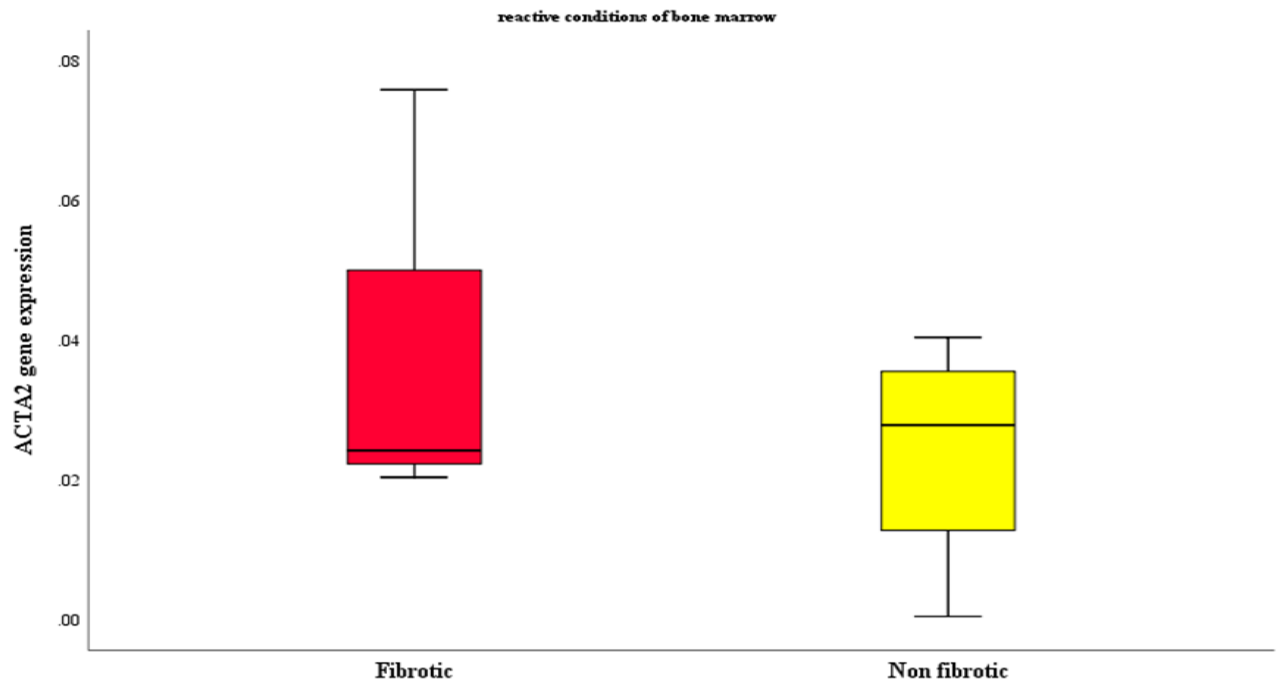

Figure 3: ACTA2 gene expression in fibrotic and non-fibrotic subgroups in reactive groups.

\section{Discussion}

Fibrosis and resultant organ failure account for at least one third of deaths worldwide. Since fibrosis is common and has adverse effects in all organs, it is an attractive therapeutic target (16).

Although we understand many of the cellular and molecular processes underlying fibrosis, there are few effective therapies and fewer that target fibrogenesis specifically. These facts highlight the need for a deeper comprehension of the pathogenesis of fibrogenesis and the translation of this knowledge to novel treatments (3).

The present study showed no statistically significant difference in ACTA2 gene expression levels in cases with bone marrow fibrosis compared to those with no bone marrow fibrosis neither in the neoplastic nor in the reactive groups.

In concordance with these results Zhao et al. addressed whether $\alpha$-SMA-expressing myofibroblasts are detectable in fibrotic muscles of $m d x 5 c v$ mice, a mouse model for Duchenne muscular dystrophy (DMD), and whether the $\alpha$-SMA expression correlates with the fibrogenic function of intramuscular fibrogenic cells. They found that $\alpha$-SMA immunostaining signal was not detected in collagen I (GFP)-expressing cells in fibrotic muscles of ColI$G F P / m d x 5 c v$ mice, but it was readily detected in smooth muscle cells lining intramuscular blood vessel walls (17). 
It was concluded that myofibroblasts are present in fibrotic skeletal muscles, but their expression of $\alpha$-SMA is not detectable by immunostaining. They also reported that the level of $\alpha$-SMA expression by intramuscular fibrogenic cells does not correlate positively with the level of collagen gene expression or the severity of skeletal muscle fibrosis in the $m d x 5 c v$ mice and they concluded that $\alpha$ SMA is not a functional marker of fibrogenic cells in skeletal muscle fibrosis associated with muscular dystrophy (17).

In contrast to our findings, Varma et al. selected 32 pairs of baseline and follow-up liver biopsies taken from stable paediatric liver transplant recipients and performed morphometric quantification of "ASMA positive area percentage" on the baseline biopsy as well as histological and fibrosis assessment using Metavir and liver allograft fibrosis scores (LAFSc) on all biopsies (18).

They reported that significant association was seen between extent of ASMA positivity on baseline biopsy and "prospective change in fibrosis" using Metavir $(\mathrm{p}$ value $=0.02)$, cumulative LAFSc ( $\mathrm{p}$ value $=0.02)$, and portal LAFSc ( $\mathrm{p}$ value $=0.01)$ values. ASMA positive area percentage $>1.05$ predicted increased fibrosis on next biopsy with $90.0 \%$ specificity. They came to the conclusion that ASMA quantification can predict the future course of fibrosis after liver transplantation (18).

Another study investigated the relationship between ASMA and type 1 collagen expression (COL1A1), a major extracellular matrix protein important in liver fibrosis with the results demonstrating that knockout of ACTA2 gene leads to reduced liver fibrosis and COL1 expression (5).

These differences can be justified in part by differences in the technique used to detect ASMA expression and the difference in the tissue used. Also in vitro culture can alter the gene expression. In the future, it will be important to carry out more genetic lineage tracing experiments of stromal cell populations to identify the source of active myofibroblasts in fibrosis and precisely characterize changes in cellular behaviour over time.

\section{Conclusion}

In summary, we have investigated whether $\alpha$-SMA plays a role in pathogenesis of bone marrow fibrosis and whether it can be used alone as a marker of pathologic fibroblasts affecting the bone marrow. Our results suggest that $\alpha$-SMA alone, cannot be 
considered an independent marker in bone marrow fibrosis and does not probably play a process-limiting role for both contractile and collagen-producing fibroblast. Exploration of related genetic pathway is recommended for this purpose.

\section{Abbreviations:}

Bone marrow (BM), myeloproliferative neoplasms (MPNs), extracellular matrix (ECM), smooth muscle alpha-actin (SM a-actin), collagen type 1A1 (COL1A1), Actin Alpha 2 (ACTA2), transforming growth factor-beta (TGF- $\beta$ ), extracellular-signalregulated kinase (ERK), acute myeloid leukemia (AML), myelodysplastic syndrome (MDS), polymerase chain reaction (PCR), complementary DNA (cDNA), liver allograft fibrosis scores (LAFSc), Duchenne muscular dystrophy (DMD).

\section{Conflict of interest:}

\section{No conflict of interest.}

\section{References:}

1. Gleitz HF, Kramann R, Schneider RK. Understanding deregulated cellular and molecular dynamics in the haematopoietic stem cell niche to develop novel therapeutics for bone marrow fibrosis. The Journal of pathology. 2018 Jun; 245(2):138-146.

2. Kuter DJ, Bain B, Mufti G, Bagg A, Hasserjian RP. Bone marrow fibrosis: pathophysiology and clinical significance of increased bone marrow stromal fibres. British journal of haematology. 2007 Nov;139(3):351-362.

3. Rockey DC, Bell PD, Hill JA. Fibrosis-a common pathway to organ injury and failure.
New England Journal of Medicine. 2015 Mar 19;372(12):1138-1149.

4. Hinz B, Phan SH, Thannickal VJ, Galli A, Bochaton-Piallat ML, Gabbiani G. The myofibroblast: one function, multiple origins. The American journal of pathology. 2007 Jun 1;170(6):1807-1816.

5. Rockey DC, Du Q, Shi Z. Smooth Muscle $\alpha$ Actin Deficiency Leads to Decreased Liver Fibrosis via Impaired Cytoskeletal Signaling in Hepatic Stellate Cells. The American journal of pathology. 2019 Nov 1;189(11):2209-2220.

6. Sandbo N, Dulin N. Actin cytoskeleton in myofibroblast differentiation: ultrastructure defining form and driving function. Translational Research. 2011 Oct 1;158(4):181196.

7. Ueyama HI, Hamada HI, Battula NA, Kakunaga TA. Structure of a human smooth muscle actin gene (aortic type) with a unique intron site. Molecular and cellular biology. 1984 Jun 1;4(6):1073-1080.

8. Goffin JM, Pittet P, Csucs G, Lussi JW, Meister JJ, Hinz B. Focal adhesion size controls tension-dependent recruitment of $\alpha$-smooth muscle actin to stress fibers. The Journal of cell biology. 2006 Jan 16;172(2):259-268.

9. Rockey DC, Weymouth N, Shi Z. Smooth muscle $\alpha$ actin (Acta2) and myofibroblast function during hepatic wound healing. PloS one. 2013;8(10).

10. Harburger DS, Calderwood DA. Integrin signalling at a glance. Journal of cell science. 2009 Jan 15;122(2):159-163.

11. Shi Z, Rockey DC. Upregulation of the actin cytoskeleton via myocardin leads to increased expression of type 1 collagen. Laboratory Investigation. 2017 Dec;97(12):1412-1426. 
12. Takeji M, Moriyama T, Oseto S, Kawada N, Hori M, Imai E, Miwa T. Smooth muscle $\alpha$ actin deficiency in myofibroblasts leads to enhanced renal tissue fibrosis. Journal of Biological Chemistry. 2006 Dec 29;281(52):40193-40200.

13. Bain B J, Clark D M, Lampert I A, et al., (2001): Bone marrow pathology, 2nd Edition. London; Blackwell Science Ltd: pp601-609.

14. Peterson LC, Brunning RD. Bone marrow specimen processing. Neoplastic hematopathology. Philadelphia: Lippincott Williams and Wilkins. 2001;1391.

15. Kvasnicka HM, Beham-Schmid C, Bob R, Dirnhofer S, Hussein K, Kreipe H, Kremer M, Schmitt-Graeff A, Schwarz S, Thiele J, Werner M. Problems and pitfalls in grading of bone marrow fibrosis, collagen deposition and osteosclerosis-a consensus-based study. Histopathology. 2016 May;68(6):905-915.

16. Zeisberg M, Kalluri R. Cellular mechanisms of tissue fibrosis. 1. Common and organ-specific mechanisms associated with tissue fibrosis. American Journal of Physiology-Cell Physiology. 2013 Feb 1;304(3):C216-225.

17. Zhao W, Wang X, Sun KH, Zhou L. $\alpha$-smooth muscle actin is not a marker of fibrogenic cell activity in skeletal muscle fibrosis. PloS one. 2018;13(1).

18. Varma S, Stéphenne X, Komuta M, Bouzin C, Ambroise J, Smets F, et al., To Evaluate Significance of Alpha Smooth Muscle Actin (ASMA) Expression on Liver Biopsy as Predictor of Future Graft Fibrosis in Pediatric Liver Transplant (LT) Recipients. Journal of Clinical and Experimental Hepatology. 2016 Jul $1 ; 6:$ S61.

To cite this article: Amira Osama, Amal El-Mahdy, Samea Rizk, Howayda Mohamed, Hanan El-Hosseiny, Manal Wilson , Amira Noureldin. Alpha-Smooth muscle actin $(\alpha-$ SMA) gene, a possible derive of myofibroblasts in bone marrow fibrosis, BMFJ, 2020; 37(2):439-448. DOI: 10.21608/bmfj.2020.24976.1221 